\title{
Experimental Study Using Multiple Strains of Prion Disease in Cattle Reveals an Inverse Relationship between Incubation Time and Misfolded Prion Accumulation, Neuroinflammation, and Autophagy
}

\author{
Najiba Mammadova, ${ }^{* \dagger \ddagger}$ M. Heather West Greenlee, ${ }^{\dagger \dagger \S}$ S. Jo Moore, ${ }^{\llbracket}$ Donald S. Sakaguchi, ${ }^{\star \ddagger}$ and Justin J. Greenlee
}

\begin{abstract}
From the Department of Genetics, Development and Cell Biology, ${ }^{*}$ the Immunobiology Graduate Program, ${ }^{\dagger}$ and the Neuroscience Graduate Program, ${ }^{\ddagger}$ Iowa State University, Ames; the Department of Biomedical Sciences, ${ }^{\S}$ Iowa State University College of Veterinary Medicine, Ames; and the Virus and Prion Research Unit, ${ }^{\top}$ National Animal Disease Center, US Department of Agriculture, Agricultural Research Service, Ames, Iowa
\end{abstract}

\author{
Accepted for publication \\ March 20, 2020. \\ Address correspondence to $\mathrm{M}$. \\ Heather West Greenlee, Ph.D. \\ Department of Biomedical Sci- \\ ences, Iowa State University \\ College of Veterinary Medi- \\ cine, 2008 Vet Med, 1800 \\ Christensen Dr., Ames, IA \\ 50011-1250. E-mail: \\ mheather@iastate.edu.
}

\begin{abstract}
Proteinopathies result from aberrant folding and accumulation of specific proteins. Currently, there is a lack of knowledge about the factors that influence disease progression, making this a key challenge for the development of therapies for proteinopathies. Because of the similarities between transmissible spongiform encephalopathies (TSEs) and other protein misfolding diseases, TSEs can be used to understand other proteinopathies. Bovine spongiform encephalopathy (BSE) is a TSE that occurs in cattle and can be subdivided into three strains: classic BSE and atypical BSEs ( $H$ and $L$ types) that have shorter incubation periods. The NACHT, LRR, and PYD domains-containing protein 3 inflammasome is a critical component of the innate immune system that leads to release of IL-1 $\beta$. Macroautophagy is an intracellular mechanism that plays an essential role in protein clearance. In this study, the retina was used as a model to investigate the relationship between disease incubation period, prion protein accumulation, neuroinflammation, and changes in macroautophagy. We demonstrate that atypical BSEs present with increased prion protein accumulation, neuroinflammation, and decreased autophagy. This work suggests a relationship between disease time course, neuroinflammation, and the autophagic stress response, and may help identify novel therapeutic biomarkers that can delay or prevent the progression of proteinopathies. (Am J Pathol 2020, 190: 1461-1473; https://doi.org/10.1016/j.ajpath.2020.03.006)
\end{abstract}

Many neurodegenerative diseases, including Parkinson disease (PD), Alzheimer disease (AD), Huntington disease, and transmissible spongiform encephalopathies (TSEs), result from protein misfolding, followed by the subsequent aggregation of these proteins into abnormal, toxic species that accumulate in tissues. ${ }^{1-3}$ A key feature that distinguishes prion diseases from other protein misfolding diseases is the transmissibility of TSE agents. Although the causative agent of all TSEs is misfolded prion protein $\left(\mathrm{PrP}^{\mathrm{Sc}}\right)$, different strains of TSEs within a species may be distinguished by different pathogenesis and disease phenotypes. These phenotypes include distinct immunohistologic characteristics (ie, lesion profiles and neuroanatomic deposition patterns of $\operatorname{PrP}^{\mathrm{Sc}}$ ), clinical signs, and incubation periods. $^{2,4-11}$ Specifically, bovine spongiform encephalopathy (BSE) can be subdivided into classic BSE, colloquially known as mad cow disease, and sporadic/ atypical BSE. ${ }^{4}$ Atypical BSE can be further subdivided into $\mathrm{H}$ and $\mathrm{L}$ types, with the $\mathrm{H}$ and $\mathrm{L}$ designations based on a higher or lower molecular mass, respectively, of the unglycosylated $\mathrm{PrP}^{\mathrm{Sc}}$ isoform, when compared with classic BSE. ${ }^{4}$ Both H- and L-type BSE, herein referred to as BSE-H

\footnotetext{
Supported by congressionally appropriated funds (US Department of Agriculture, Agricultural Research Service).

Disclosures: None declared.

Presented in part as an abstract at PRION 2019, May 21 to 24, 2019, Edmonton, AB, Canada.

Current address of S.J.M., Department of Pathology, Animal and Plant Health Agency, Department for Environment Food and Rural Affairs, Addelstone, Surrey, United Kingdom.
} 
and BSE-L, respectively, have shorter incubation periods after experimental intracranial inoculation and, therefore, an accelerated disease progression when compared with classic BSE. One of the key challenges for drug discovery and development of therapeutic approaches is a lack of wellvalidated disease biomarkers because of a limited understanding of the molecular and cellular factors that interact with the misfolded proteins and influence how rapidly a disease progresses. ${ }^{1}$ Because of the striking parallels of TSEs to the noninfectious protein misfolding diseases (ie, PD, AD, and Huntington disease), prion diseases can be used to study and understand other proteinopathies. ${ }^{1}$ In this study, we exploited the differences between classic and atypical BSEs to identify the molecular factors or determinants that are associated with the slower progression of classic BSE, versus the more rapid progression of atypical BSEs. Specifically, the strain-dependent variation in incubation periods between classic and atypical BSEs was used as a controlled framework to examine the relationship between a diverse set of immunologic characteristics.

$\mathrm{PrP}^{\mathrm{Sc}}$ accumulates in retinas of TSE infected animals, ${ }^{12-20}$ and antemortem changes in retinal function and morphology are detectable in BSE inoculated cattle up to 11 months before the appearance of any other clinical signs of disease, suggesting that the retina is an excellent experimental model to investigate disease pathogenesis. Accumulation of $\mathrm{PrP}^{\mathrm{Sc}}$ has also been reported in other structures of the visual system, including the optic nerve, extraocular muscle, choroid, vitreous, lens, and sclera. ${ }^{20}$ Herein, the retina was used as an experimental model to investigate the effect of $\mathrm{PrP}^{\mathrm{Sc}}$ accumulation and specific neuroinflammatory processes [including glial cell activation, NACHT, LRR, and PYD domains-containing protein 3 (NLRP3) inflammasome activation, and macroautophagy] on disease incubation periods. The NLRP3 inflammasome is a multiprotein complex that is an important component of innate immunity and a known contributor to the progression of several protein misfolding diseases (ie, TSEs, PD, and AD). ${ }^{21,22}$ Macroautophagy, often referred to as autophagy, is a highly conserved metabolic process responsible for the clearance of bulk cytoplasmic components, including protein aggregates. Several studies indicate that misfolded proteins provoke microglia-driven NLRP3 inflammasome activation that has been reported to be associated with autophagic dysfunction (ie, a decline in autophagic processes). ${ }^{21,23-27}$ However, the collective effect of inflammasome activation and autophagy on disease progression and incubation periods is unknown.

In this study, we report that disease incubation period is inversely correlated to $\operatorname{PrP}^{\mathrm{Sc}}$ accumulation, glial cell activation, and NLRP3 inflammasome activation in the retina, brainstem, and thalamus. Specifically, cattle inoculated with atypical BSE-H and BSE-L, characterized by shorter incubation periods compared with classic BSE, had greater $\mathrm{PrP}^{\mathrm{Sc}}$ deposition, robust activation of Müller glia, astrocytes, and microglia, and microglia-driven NLRP3 inflammasome activation. Our results show that incubation period accompanies an inverse correlation between $\mathrm{PrP}^{\mathrm{Sc}}$ associated NLRP3 inflammasome activation and autophagy. Specifically, we describe opposing events in the autophagic pathway: up-regulation of autophagy detected in cattle inoculated with classic BSE; and a decline in autophagy in cattle inoculated with BSE-H or BSE-L that paralleled greater $\mathrm{PrP}^{\mathrm{Sc}}$ accumulation, neuroinflammation, and shorter incubation periods. We demonstrate an association between incubation period and several molecular disease processes (ie, accumulation of misfolded proteins, glial cell activation, NLRP3 inflammasome activation, and macroautophagy) in vivo, which can further our understanding of the factors that influence disease progression. This study provides a better knowledge of the molecular basis of accelerated disease progression in different strains of prion disease. Because of the similarity of TSEs with other protein misfolding diseases, this work could have important implications for understanding the interplay between misfolded proteins and the cellular responses in vivo and identifying biomarkers that can be targeted for clinical trials.

\section{Materials and Methods}

\section{Ethics Statement}

This experiment was performed in accordance with NIH's Guide for the Care and Use of Laboratory Animals ${ }^{28}$ and the Guide for the Care and Use of Agricultural Animals in Research and Teaching (Federation of Animal Science Societies, Champaign, IL). The protocol was approved by the Institutional Animal Care and Use Committee at the National Animal Disease Center (protocol number 3985) and Iowa State University (protocol number 7154).

\section{Animals and Inoculum}

Adult Holstein steers (aged 3.8 to 4.5 years) were inoculated intracranially with $1 \mathrm{~mL}$ of a $10 \%$ (wt./vol) brain homogenate, as previously described. ${ }^{29}$ Twelve cattle were inoculated with the agent of classic $\mathrm{BSE},{ }^{30}$ nine cattle were inoculated with the agent of BSE-H ${ }^{30}$ and 10 cattle were inoculated with the agent of BSE-L. ${ }^{31}$ Control cattle $(n=5)$ for this study were sham inoculated. ${ }^{32,33}$ The inoculated cattle were observed daily by animal care staff at National Animal Disease Center (NADC) and examined regularly by investigators to determine the onset of clinical disease. Cattle were euthanized at the onset of unequivocal signs of clinical disease, but were not allowed to develop severe, end-stage disease. On euthanasia, all cattle were confirmed positive by Western blot analysis or enzyme immunoassay, as directed (HerdChek BSE-Scrapie Ag Test, IDEXX, Westbrook, ME). Tissue collection was performed, as previously described. ${ }^{16}$ Brain (cerebral cortex, cerebellum, midbrain, including superior colliculus, and brainstem, including obex) and eyes were collected into $10 \%$ buffered 
formalin (eye globes were fixed in Bouin's fixative) and embedded in paraffin wax for sectioning.

\section{Immunohistochemistry and Western Blot Analysis}

Paraffin-embedded sections (4 $\mu \mathrm{m}$ thick) were rehydrated using xylene, followed by a decreasing ethanol concentration gradient $(100 \%, 90 \%$, and $70 \%)$, and a final wash with deionized $\mathrm{H}_{2} \mathrm{O}$. Heat-mediated antigen retrieval was performed using EDTA buffer ( $10 \mathrm{mmol} / \mathrm{L}$ Trizma base, $1 \mathrm{mmol} / \mathrm{L}$ EDTA solution, and $0.05 \%$ Tween $20, \mathrm{pH} 9.0$ ) or citrate buffer (ScyTek Laboratories, Logan, UT) in an autoclave for $30 \mathrm{mi}-$ nutes. Tissues were incubated with Background Buster (Innovex Biosciences Inc., Richmond, CA) for 1.5 hours. Primary antibodies against glial fibrillary acidic protein (GFAP; 1:1000; Dako, Carpinteria, CA), Ibal (1:2000; Abcam, Cambridge, UK), NLRP3 (1:250; Adipogen, San Diego, CA), apoptosis-associated speck-like protein containing a CARD (ASC; 1:500; Adipogen), caspase-1 (1:1000; Adipogen), IL-1ß (1:1000; Abcam), LC3A/B (1:2000; Cell Signaling, Danvers, MA), or caspase-3 (Santa Cruz Biotechnology, Dallas, TX) were diluted in blocking solution containing $0.1 \%$ bovine serum albumin (Sigma-Aldrich, St. Louis, MO), 0.05\% Triton X-100 (Thermo Fisher Scientific, Inc., Rockford, IL), and 5\% normal serum (Jackson ImmunoResearch, West Grove, PA) in Tris-buffered saline and incubated for 48 hours at room temperature. Tissues were washed with Tris-buffered saline and Tween $20(6 \times 5$ minutes $)$ and incubated with a secondary antibody cocktail, including $\mathrm{Cy}^{\mathrm{TM}_{3}}$ and/or Alexa Fluor 488-conjugated AffiniPure secondary antibodies (1:250; Jackson ImmunoResearch) and DAPI ( $4 \mu \mathrm{g} / \mathrm{mL}$; Sigma-Aldrich) for 1.5 hours. Following another wash, slides were mounted with Vectashield antifade mounting medium (Vector Laboratories Inc., Burlingame, CA). Negative controls were processed in parallel by omission of the primary and/or secondary antibody. For detection of $\mathrm{PrP}^{\mathrm{Sc}}$, slides were stained by an automated immunohistochemical method, described previously. ${ }^{15,29}$ The primary antibody used was F99/97.6.1 (O'Rourke, Pullman, WA) at a concentration of $5 \mu \mathrm{g} / \mathrm{mL}$.

The protein extraction method for Western blot analysis has been previously described by Mammadova et al (2019). ${ }^{34}$ The primary antibodies directed against LC3II (1:2500; Cell Signaling), pULK $1^{\mathrm{S} 757}$ (1:1000; Cell Signaling), ULK1 (1:1500; Cell Signaling), cleaved caspase-1 (p20; 1:1000; Adipogen), and cleaved IL-1 $\beta$ (p17; 1:2000; Abcam) were incubated overnight at $4^{\circ} \mathrm{C}$. All groups are normalized to control and/or mock-treated animals, providing a representative of a fold change from the control; therefore, $y$-axis units are arbitrary. Data are representative of three biological replicates.

\section{Fluorescent Microscopy and Statistical Analysis}

Retinas and brain regions of 12 cattle inoculated with the agent of classic BSE, 9 cattle inoculated with the agent of BSE-H, 10 cattle inoculated with the agent of BSE-L, and 5 shaminoculated cattle were analyzed. Fluorescence and confocal images were taken at $\times 20$ and $\times 60$ magnification, using a Nikon A1R+ Resonant Scanning Confocal System with a TiE inverted microscope and laser lines 405, 488, 561, and 640 $\mathrm{nm}$ (Nikon Instruments Inc., Melville, NY). Micrographs were generated using a commercial photo-editing system [Adobe Photoshop and Adobe Illustrator (CC); Adobe Systems, San Jose, CA]. For quantification of GFAP, Iba1, NLRP3, and ASC immunoreactivity, the percentage of the total image area limited to a threshold (outer limiting membrane to inner limiting membrane of the central retina) was analyzed using Image J (W.S. Rasband, ImageJ version 1.49, NIH, Bethesda, MD; ImageJ.net version $1.52 \mathrm{~s}$, last accessed January 15, 2019), as previously described. ${ }^{35}$ When comparing multiple specimens, identical thresholding tool settings (ie, image acquisition settings and exposure times) were used. For each animal, three different sites of the retina were analyzed to gauge for differences in immunolabeling: central retina at the thickest section of the retina adjacent to the optic nerve head and two peripheral retinal locations on opposite sides of the retina within one $\times 20$ field of view from the peripheral margin. Preliminary analyses showed no significant difference among these sites; therefore, data from the peripheral and central retinas were pooled. For each animal, the central retina, near the optic disc, within a cross-section is represented in the micrographs. For quantification of GFAP, Iba1, NLRP3, and ASC immunoreactivity, three consecutive retinal sections $(12 \mu \mathrm{m}$ thick) were analyzed, and the means \pm SEM were reported. Equivalent regions of each brain structure [ie, brainstem at the level of the obex, thalamus (interthalamic nuclei), and caudate nuclei] were used for the generation of micrographs and quantification using ImageJ. Quantified histologic data were analyzed using a two-way analysis of variance, with a Tukey's multiple comparisons test (post hoc). Prism version 6.01 for Windows (GraphPad Software, San Diego, CA) was used for statistical analysis.

\section{Results}

To investigate the relationship between incubation period and disease-associated pathology, a controlled comparison of tissues from cattle experimentally inoculated with classic and atypical BSEs (BSE-H and BSE-L) was conducted. Incubation period for classic BSE, BSE-H, or BSE-L is reported as the time from intracranial inoculation to the time when unequivocal signs of clinical disease are present. Clinical signs of disease included abnormalities in gait and/ or stance, moderate to severe ataxia, and hyperreaction to stimuli (ie, noise and movement). Cattle inoculated with atypical BSEs had significantly shorter incubation periods compared with cattle inoculated with classic BSE (Figure 1). The average incubation period for cattle inoculated with classic BSE, BSE-H, or BSE-L was $683.7 \pm 45.2$, $513.7 \pm 4.8$, and $472.8 \pm 11.9$ days postinoculation, 


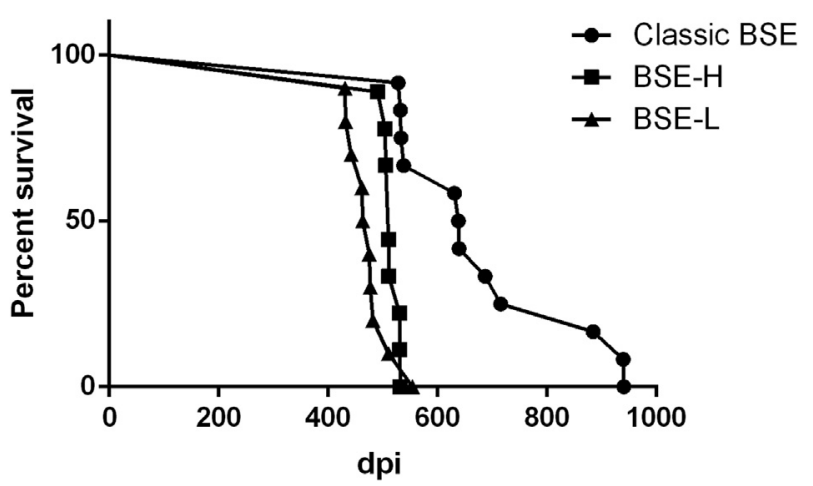

Figure 1 Incubation periods of classic and atypical bovine spongiform encephalopathies (BSEs). Kaplan-Meier survival curve showing the average incubation period, defined as the number of days from inoculation to the onset of unequivocal clinical signs for cattle inoculated with the classic BSE, BSE-H, or BSE-L agents. Data are expressed as means \pm SEM. $n=12$ classic BSE group; $n=9$ BSE-H group; $n=10 \mathrm{BSE}-\mathrm{L}$ group. dpi, days post inoculation.

respectively (Figure 1). The differences in incubation period were statistically significant $(P<0.0001)$. The migration pattern of $\operatorname{PrP}^{\mathrm{Sc}}$ on Western blot analysis was analyzed to verify that the cattle inoculated with classic or atypical BSEs retained a classic, high-type, or low-type migration pattern, as previously described. ${ }^{13,31}$

\section{Increased $\operatorname{PrP}^{\mathrm{Sc}}$ Deposition Coincides with Shorter Incubation Periods of BSE-H and BSE-L}

To investigate the relationship between $\operatorname{PrP}^{\mathrm{Sc}}$ deposition and incubation period, $\mathrm{PrP}^{\mathrm{Sc}}$ immunoreactivity was assessed in retinas of cattle inoculated with classic BSE, BSE-H, and BSE-L. Retinas from cattle inoculated with classic BSE had punctate or granular $\mathrm{PrP}^{\mathrm{Sc}}$ localized primarily to the synaptic layers (inner plexiform layer and outer plexiform layer) (Figure 2, $\mathrm{A}$ and $\mathrm{B}$ ). The distribution of $\mathrm{PrP}^{\mathrm{Sc}}$ labeling was more extensive in retinas of cattle inoculated with BSE-H or BSE-L (Figure 2, C and D). Areas of $\operatorname{PrP}^{\mathrm{Sc}}$ immunolabeling were much more intense and uniform in the synaptic layers (Figure 2, C and D). Globular deposits of $\mathrm{PrP}^{\mathrm{Sc}}$ were evident in the inner and outer segments of photoreceptor cells and dispersed throughout the nuclear layers, with robust intracellular $\mathrm{PrP}^{\mathrm{Sc}}$ immunoreactivity evident within cells located in the ganglion cell layer (Figure 2C). Compared with retinas of cattle inoculated with $\mathrm{BSE}-\mathrm{H}, \mathrm{PrP}^{\mathrm{Sc}}$ was rarely detected in the outer segments of photoreceptor cells, outer nuclear layer, and ganglion cell layer of BSE-L inoculated cattle (Figure 2, C and D). These results demonstrate that increased immunolabeling of $\mathrm{PrP}^{\mathrm{Sc}}$ in the retina correlates with shorter incubation periods, characteristic of atypical BSEs. To investigate the relationship between $\operatorname{PrP}^{\mathrm{Sc}}$ deposition and incubation period in the brain, $\mathrm{PrP}^{\mathrm{Sc}}$ immunoreactivity was assessed in the hypoglossal nuclei of the brainstem at the level of the obex, interthalamic nuclei, and caudate nuclei of cattle inoculated with classic BSE, BSE-H, and BSE-L. Compared with control, the brainstems at the level of the obex of cattle inoculated with classic BSE had aggregated $\operatorname{PrP}^{\mathrm{Sc}}$ deposits (Figure 2, E and F). Compared with cattle with classic BSE, $\mathrm{PrP}^{\mathrm{Sc}}$ immunolabeling in the brainstems of cattle inoculated with BSE-H or BSE-L was more intense (Figure 2, G and $\mathrm{H})$. A similar trend in $\mathrm{PrP}^{\mathrm{Sc}}$ deposition was seen in the thalami of these cattle (Figure 2, I-L). Conversely, $\operatorname{PrP}^{\mathrm{Sc}}$ immunolabeling in the caudate nuclei of cattle inoculated with classic BSE, BSE-H, or BSE-L was similar in the deposition pattern (ie, aggregated and plaque-like $\operatorname{PrP}^{\mathrm{Sc}}$ deposits) and area of immunolabeling (Figure 2, M-P). Strain-dependent differences in incubation time of classic, H-type, and L-type BSE did not parallel differences in the amount of $\operatorname{PrP}^{\mathrm{Sc}}$ accumulation in the caudate nuclei. These results demonstrate that increased area of $\mathrm{PrP}^{\mathrm{Sc}}$ deposition in some brain regions (ie, brainstem and thalamus) correlates with shorter incubation times of atypical BSEs.

\section{Glial Cell Activation Parallels PrP ${ }^{\mathrm{Sc}}$ Accumulation}

Activation of Müller glia and microglia is a widely reported retinal response during progression of TSEs, like many protein misfolding diseases. ${ }^{12,13,15,36}$ To assess the activation of these principal retinal glial cells, the distribution of GFAP and Iba1 immunoreactivity, respectively, was measured. GFAP immunoreactivity in quiescent Müller glia is constrained to their end feet. However, under conditions of retinal injury or stress, GFAP expression is increased and widely distributed throughout the radial processes of Müller glia that span from the inner limiting membrane to the outer limiting membrane.$^{37}$ GFAP immunoreactivity in the retinas of control cattle was localized to the Müller glial end feet and astrocytes in the optic fiber layer (Figure 3A). Compared with control cattle, the retinas of cattle inoculated with classic BSE had increased GFAP immunoreactivity, observed in the radial processes spanning the retina from the optic fiber layer to the outer plexiform layer (Figure 3B). When quantified, there was a significant difference in GFAP immunoreactivity between the retinas from cattle inoculated with sham and classic BSE $(P>0.0001)$ (Figure 3I). Compared with the retinas of cattle inoculated with classic BSE, the retinas from cattle inoculated with BSE-H or BSE-L had robust GFAP immunoreactivity, spanning the retina from the optic fiber layer, with hypertrophied Müller glial end feet and activated astrocytes, to the outer limiting membrane (Figure 3, B-D). When quantified, GFAP immunoreactivity in retinas of cattle inoculated with BSE-H and BSE-L was significantly greater than that of classic BSE $(P<0.01$ and $P<0.05$, respectively) (Figure 3I).

An antibody against Iba1, microglia/macrophage specific calcium-binding protein, was used to assess activation of microglia in the retinas of all cattle. Assessment of Iba1 immunoreactivity enables the analysis of cell shape and morphologic changes of microglia and provides information as to their function (ie, routine surveillance or phagocytosis). Compared with control, retinas of cattle inoculated 

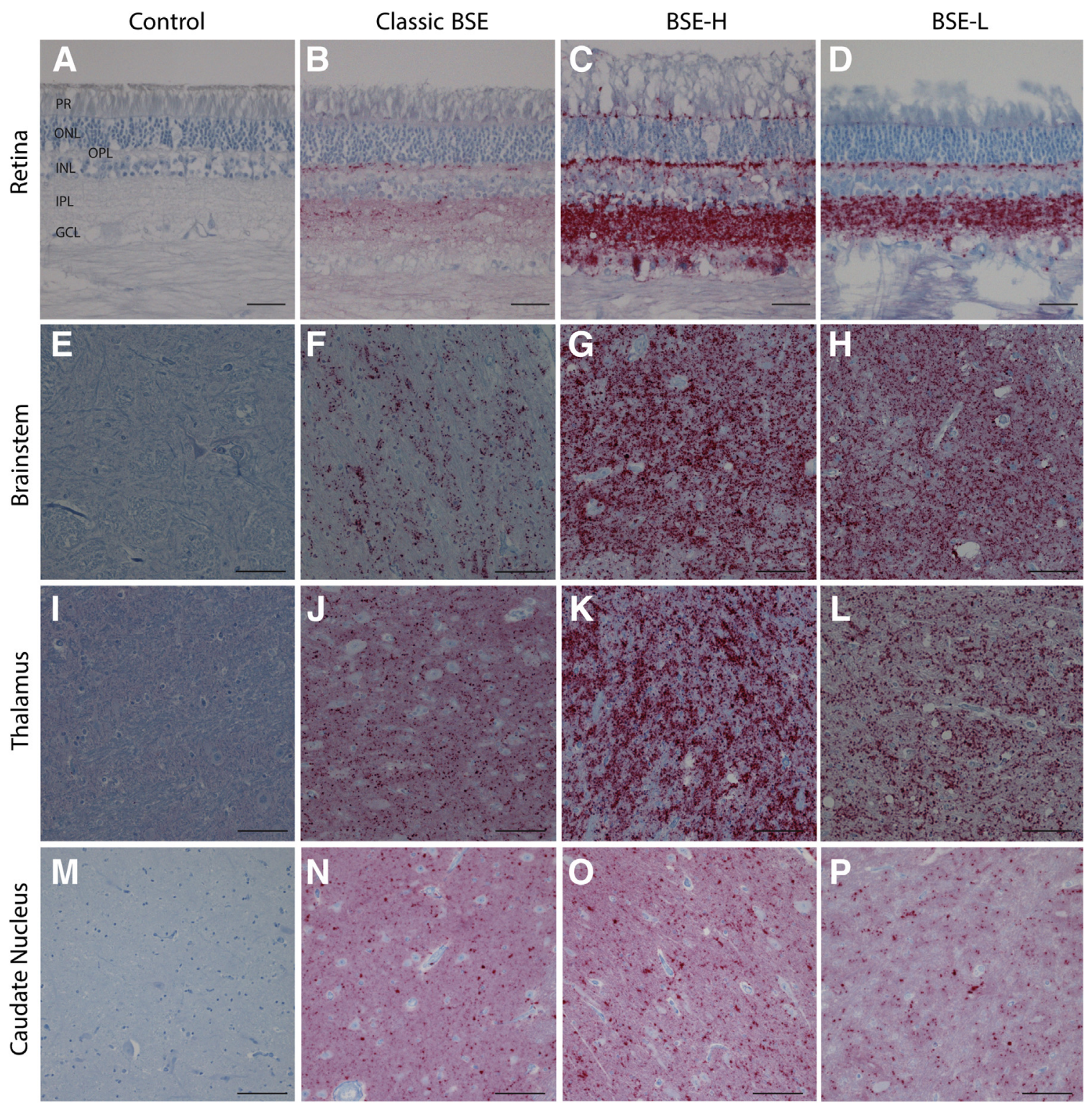

Figure 2 Accumulation of misfolded prion protein ( $\mathrm{PrP}^{\mathrm{Sc}}$ ) in tissues from cattle with classic bovine spongiform encephalopathy (BSE), BSE-H, or BSE-L. A: The retinas from negative control cattle have no PrPSc immunoreactivity. B: The retinas from cattle inoculated with classic BSE had PrPSc immunoreactivity localize to the outer plexiform layer (OPL) and inner plexiform layer (IPL). C and D: The retinas from cattle inoculated with BSE-H or BSE-L have increased PrPSc immunoreactivity in all retinal layers. E-H: Brainstem at the level of the obex (hypoglossal nucleus) from cattle inoculated with BSE-H or BSE-L has more PrPSc immunoreactivity compared with cattle inoculated with classic BSE. I-L: Thalamus (interthalamic nuclei) from cattle inoculated with BSE-H or BSE-L has more $\mathrm{PrP}^{\mathrm{SC}}$ immunoreactivity compared with that from cattle inoculated with classic BSE. M-P: PrP ${ }^{S C}$ immunoreactivity in the caudate nuclei from cattle inoculated with the BSE-H or BSE-L agents is comparable to classic BSE. Scale bars $=40 \mu \mathrm{m}(\mathbf{A}-\mathbf{P})$. GCL, ganglion cell layer; INL, inner nuclear layer; ONL, outer nuclear layer; PR, photoreceptor layer.

with classic BSE had Iba1 immunoreactive microglia with small somata and thin/long processes, sparsely dispersed through the synaptic layers (Figure 3, E and F). Quantification of Ibal immunoreactivity showed no significant difference between retinas of control cattle and those inoculated with classic BSE (Figure 3J). When compared with retinas from control and classic BSE inoculated cattle, the retinas from cattle inoculated with BSE-H or BSE-L had a marked increase in the distribution of Iba1 immunoreactive microglia and a corresponding change to an amoeboidlike morphology (Figure 3, E-H). Iba1 immunoreactivity in the retinas from cattle inoculated with atypical BSEs was widely distributed throughout all retinal layers, with substantially more in synaptic layers, and also was detected in the neuronal layers and the outer segments (Figure 3, G and $\mathrm{H})$. When quantified, Ibal immunoreactivity in retinas of cattle inoculated with BSE-H and BSE-L was significantly greater than that of classic BSE $(P<0.0001)$ (Figure $3 \mathrm{~J})$.

To assess the activation of astrocytes and microglia in the brain (ie, brainstem at the level of the obex, thalamus, and caudate nucleus), the distribution and extent of GFAP and Iba1 immunolabeling, respectively, was measured 

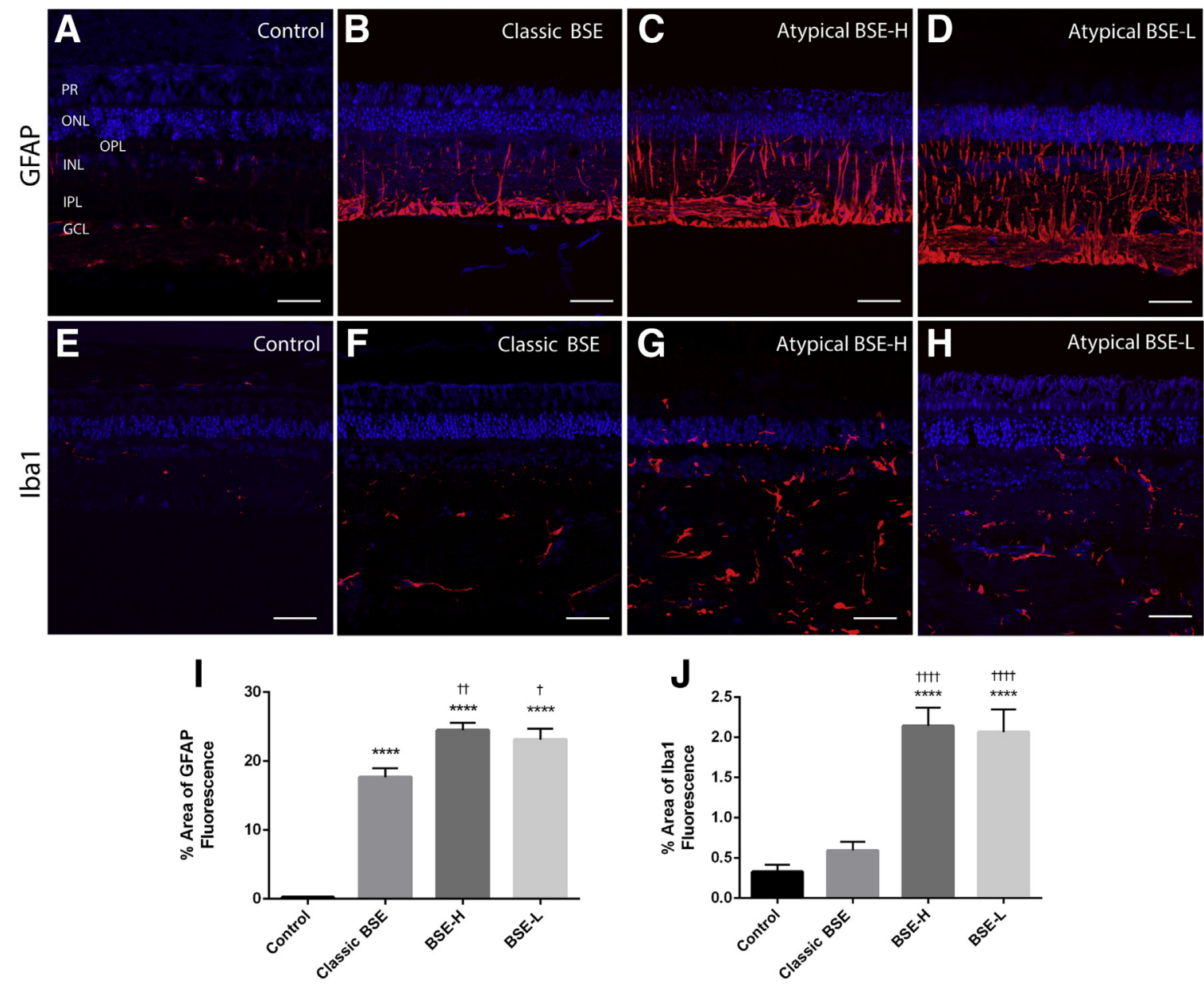

Figure 3 Activation of retinal glial cells. A: Glial fibrillary acidic protein (GFAP) is localized to end feet of Müller glia in retinas of control cattle. B: Distribution of GFAP immunoreactivity is increased in the retinas of cattle with classic bovine spongiform encephalopathy (BSE). Immunoreactivity spans the retina from the ganglion cell layer (GCL) to the outer plexiform layer (OPL). C and D: The retinas from cattle inoculated with BSE-H or BSE-L have significantly increased GFAP immunoreactivity, spanning the entire retinal thickness. $\mathbf{E}$ and F: Iba-1 immunolabeled microglia in the retinas from cattle inoculated with classic BSE are quiescent, with long processes and small cell bodies that are localized primarily to the plexiform layers. $\mathbf{G}$ and $\mathbf{H}$ : Robust microglial activation is evident in retinas of BSE-H or BSE-L inoculated cattle. Iba1-positive microglia have larger cell bodies, have thicker/retracted processes, and are localized to the synaptic and nuclear layers. I and J: Bar graphs show significant increase in GFAP and Iba1 immunoreactivity in retinas of cattle inoculated with classic BSE, BSE-H, and BSE-L. Data are expressed as means \pm SEM (I and J). ${ }^{* * * * P} P 0.0001$ versus control; ${ }^{\dagger} P<0.05,{ }^{\dagger \dagger} P<0.01$, and ${ }^{\dagger \dagger \dagger \dagger} P<0.0001$ versus classic BSE. Scale bars $=40 \mu \mathrm{m}(\mathbf{A}-\mathbf{H})$. INL, inner nuclear layer; IPL, inner plexiform layer; ONL, outer nuclear layer; PR, photoreceptor layer.

(Supplemental Figures S1 and S2). Compared with control, all three brain regions of cattle inoculated with classic BSE had significantly more GFAP immunoreactivity (Supplemental Figure S1). Compared with classic BSE cattle, GFAP immunoreactivity in the brainstem and thalamus of cattle inoculated with BSE-H or BSE-L was stronger (Supplemental Figure S1). Conversely, GFAP immunoreactivity in the caudate nuclei of cattle inoculated with classic BSE, BSE-H, or BSE-L was comparable in pattern and amount (Supplemental Figure S1). Similar to the retina, Iba1 immunoreactivity in all three brain regions of cattle inoculated with classic BSE was not significantly different than control (Supplemental Figure S2, M-O). Compared with classic BSE cattle, Iba1 immunoreactivity in the brainstem and thalamus of cattle inoculated with BSE-H or BSE-L was significantly higher (Supplemental Figure S2, $\mathrm{M}-\mathrm{O}$ ). Similar to $\mathrm{PrP}^{\mathrm{Sc}}$ and GFAP immunoreactivity, Iba1 immunoreactivity in the caudate nuclei of cattle inoculated with classic BSE, BSE-H, or BSE-L was comparable (Supplemental Figure S2O).

Taken together, these results demonstrate that compared with classic BSE, retinas of BSE-H or BSE-L inoculated cattle had robust activation of Müller glia, astrocytes, and microglia. These observations correlated with intense $\operatorname{PrP}^{\mathrm{Sc}}$ accumulation, also detected in retinas of BSE-H or BSE-L cattle. Similar differences between classic and atypical BSEs were observed in the brainstem and thalamus. However, activation of astrocytes and microglia in the caudate nucleus was comparable in cattle inoculated with all three strains of BSE and paralleled levels of $\operatorname{PrP}^{\mathrm{Sc}}$ accumulation in the tissue. Thus, these results collectively suggest a relationship between shorter incubation times, greater accumulation of $\operatorname{PrP}^{\mathrm{Sc}}$, and evidence of neuroinflammation. 

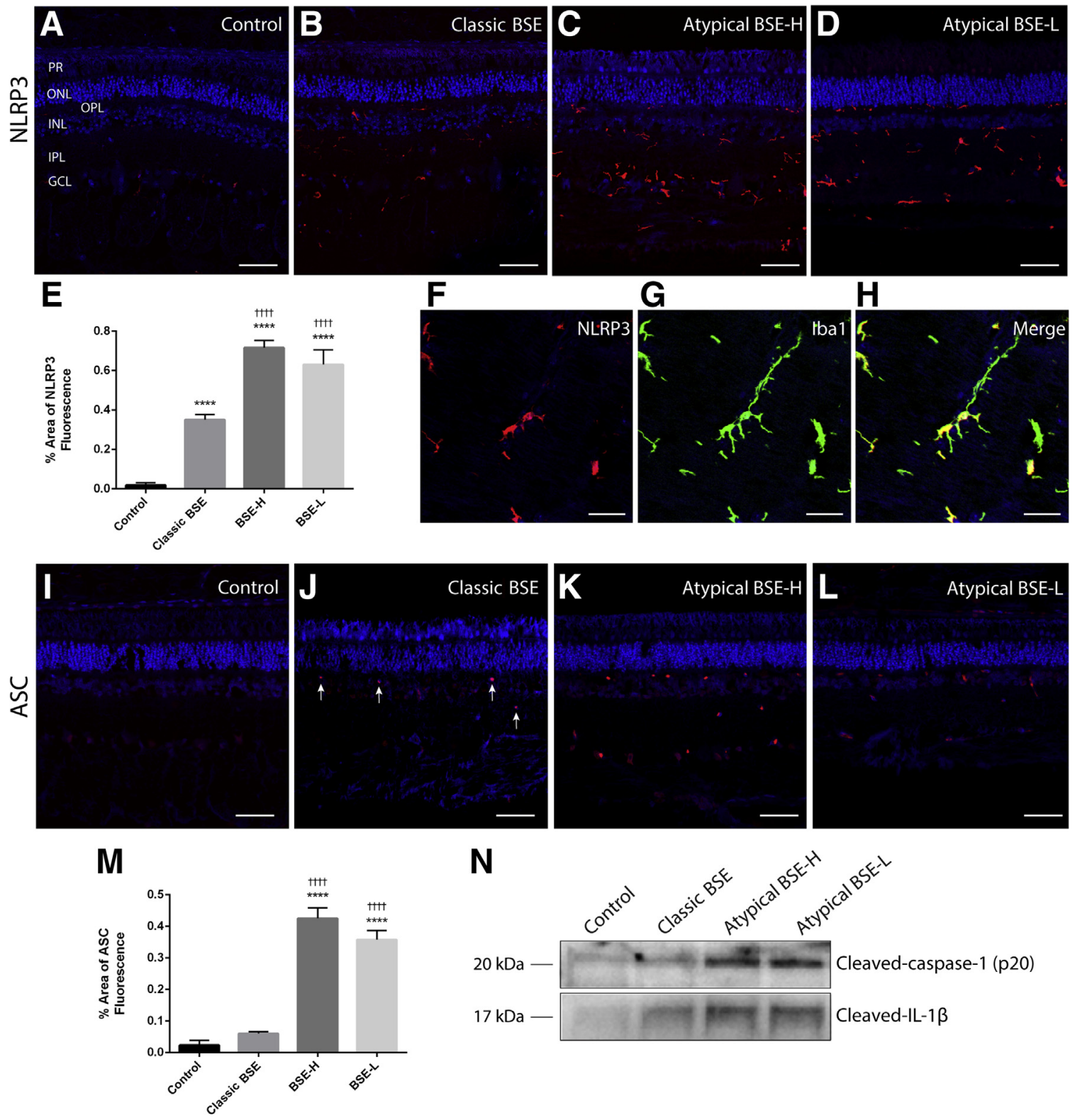

$\mathbf{N}$
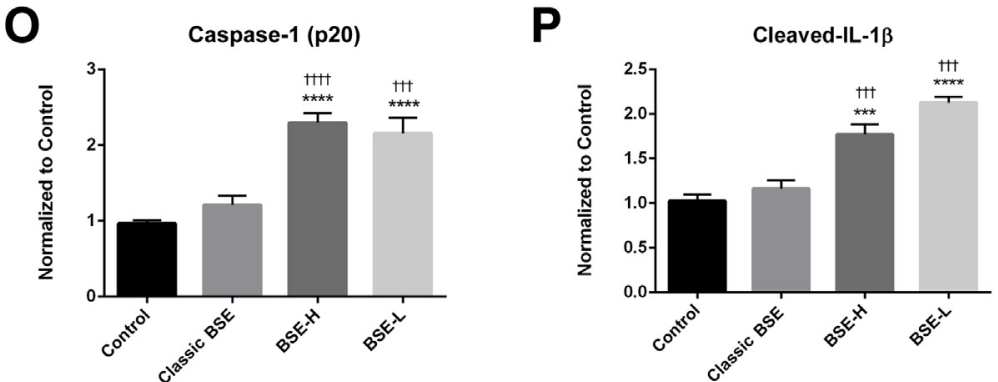

Figure 4 NALP3 (also known as NLRP3) inflammasome activation. A-M: Immunohistochemical analysis of key inflammasome components that are upregulated in cattle inoculated with bovine spongiform encephalopathy (BSE)-H or BSE-L compared with cattle inoculated with classic BSE. A-H: NLRP3. I-M: ASC. E and M: Bar graphs show percentage area of fluorescence for NLRP3 and ASC. F-H: Colocalization of Iba $1^{+}$microglia and NLRP3, confirming presence of NLRP3 inflammasome in activated, hypertrophic microglia. N: Western blot analysis of cleaved caspase-1 (p20) and cleaved IL-1 $\beta$ (p17) expression levels in the retina. To highlight the band of interest, blots were cropped from different parts of the same gel, or from different gels, with consistent exposure times. Data are not spliced together from different gels. $\mathbf{0}$ and $\mathbf{P}$ : Representative bar graphs showing quantitative densitometric analysis of cleaved caspase-1 (p20) and cleaved IL-1 $\beta$ (p17) in retinas of cattle inoculated with sham, classic BSE, BSE-H, and BSE-L. Data are expressed as means \pm SEM (E, M, $\mathbf{0}$, and P). ${ }^{* * *} P<0.0001,{ }^{* * * *} P<0.0001$ versus control; ${ }^{\dagger \dagger \dagger} P<0.001,{ }^{\dagger \dagger \dagger \dagger} P<0.0001$ versus classic BSE. Scale bars $=40 \mu \mathrm{m}(\mathbf{A}-\mathbf{D}$ and $\mathbf{F}-\mathbf{L}) . \mathrm{GCL}$, ganglion cell layer; INL, inner nuclear layer; IPL, inner plexiform layer; $\mathrm{ONL}$, outer nuclear layer; $\mathrm{OPL}$, outer plexiform layer; PR, photoreceptor layer. 

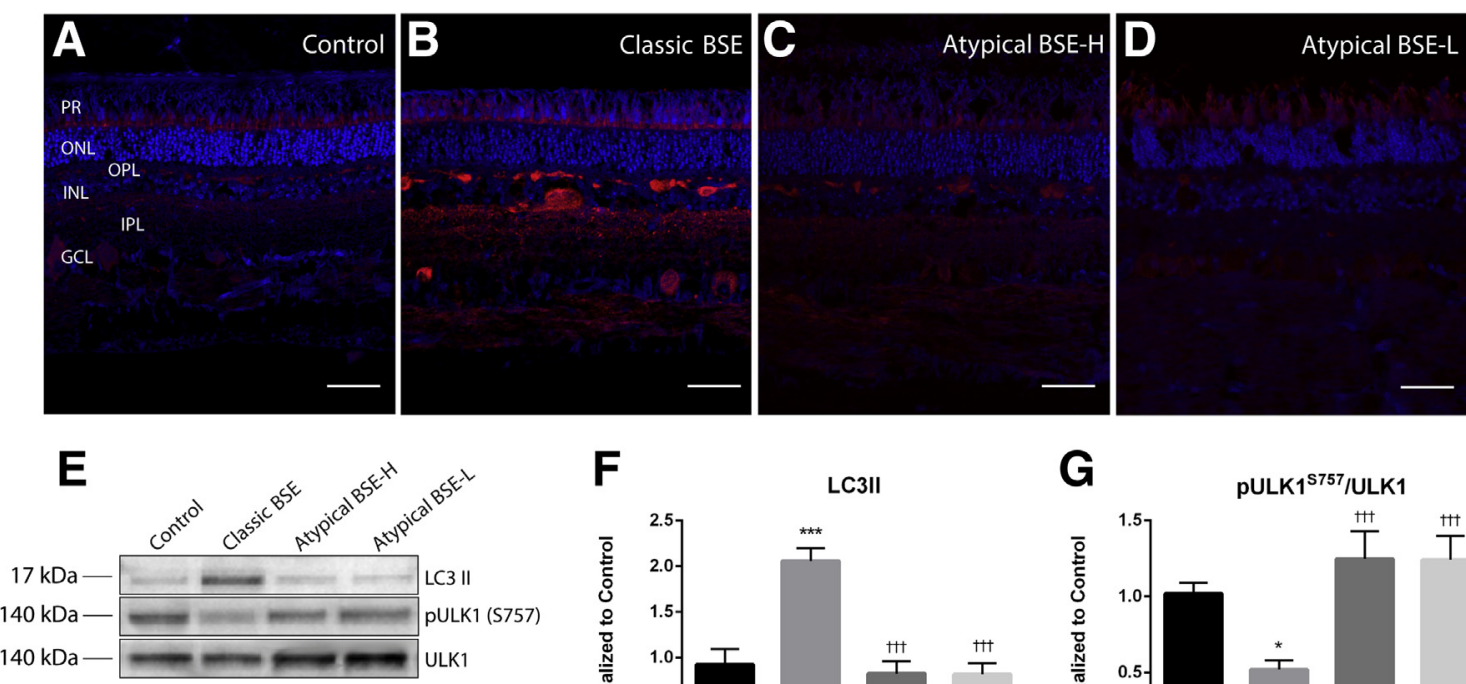

$\mathbf{F}$
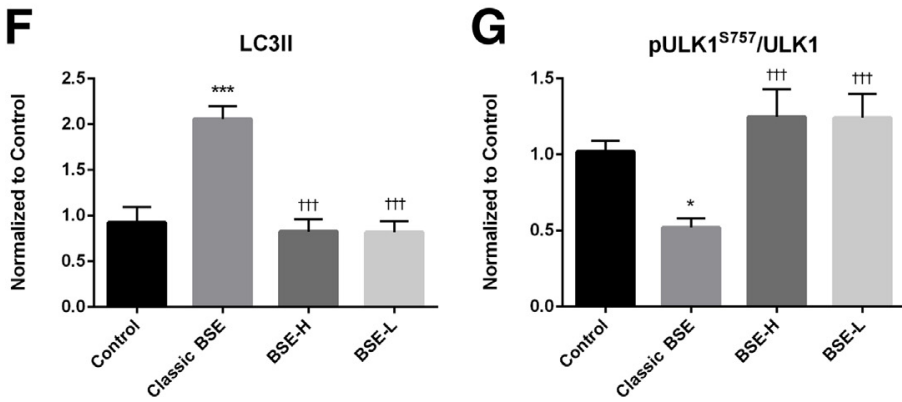

Figure 5 Strain-dependent changes in macroautophagy. A: Diffuse LC3 immunoreactivity in the retinas from negative control cattle is representative of basal autophagy. B: Increase in LC3-immunoreactive autophagosomes in outer segments of photoreceptor cells, outer and inner plexiform layers, and ganglion cell layer, evident in retinas from cattle inoculated with classic bovine spongiform encephalopathy (BSE), is suggestive of autophagic response to misfolded protein $\left(\mathrm{PrP}^{\mathrm{SC}}\right)$ accumulation. C and D: Retinas from cattle inoculated with BSE-H or BSE-L lack LC3-immunoreactive puncta similar to control retinas. E: Western blot analysis of LC3II, pULK1 ${ }^{\mathrm{S757}}$, and ULK1 expression levels in the retina. To highlight the band of interest, blots were cropped from different parts of the same gel, or from different gels, with consistent exposure times. Data are not spliced together from different gels. $\mathbf{F}$ and $\mathbf{G}$ : Representative bar graphs showing quantitative densitometric analysis of LC3II and pULK $1^{\text {S757 }} /$ ULK1. Data are expressed as means \pm SEM (F and $\left.\mathbf{G}\right)$. ${ }^{\star} P<0.05,{ }^{*} * P<0.001$ versus control; ${ }^{\dagger \dagger} P<0.001$ versus classic BSE. Scale bars $=40 \mu \mathrm{m}(\mathbf{A}-\mathbf{D}) . \mathrm{GCL}$, ganglion cell layer; INL, inner nuclear layer; IPL, inner plexiform layer; 0 NL, outer nuclear layer; OPL, outer plexiform layer; PR, photoreceptor layer.

\section{Activation of the NLRP3 Inflammasome Parallels the Shorter Incubation Times of Atypical BSE-H and BSE-L}

Contribution of NLRP3 inflammasome activation to the progression of protein misfolding diseases has been extensively described in TSEs, PD, and AD. ${ }^{22}$ To probe the relationship between incubation time and NLRP3 inflammasome activation in BSE, the expression of key inflammasome components was assessed: NLRP3, the adaptor molecule ASC, active caspase-1, and active IL-1 $\beta$ (Figure 4). NLRP3 immunoreactivity in the retinas of control cattle was negligible (Figure 4A). Retinas from cattle inoculated with classic BSE had increased NRLP3 immunoreactivity, and similar to Ibal immunoreactivity, it was localized to the synaptic layers (Figure 4B). Retinas of cattle inoculated with atypical BSEs had robust NLRP3 immunoreactivity, with approximately a twofold increase compared with classic BSE (Figure 4, C-E). Double immunofluorescence and confocal microscopy confirmed that NLRP3 was almost exclusively localized to $\mathrm{Iba}^{+}$ microglia (Figure 4, F-H), consistent with previous reports linking the detrimental effects of chronic microgliosis to inflammasome activation. ${ }^{21,23,38}$ Immunohistochemical analysis of ASC immunoreactivity yielded similar results (Figure 4, I-M). In contrast to NLRP3 expression, however, negligible multifocal ASC immunoreactivity was observed in the retinas from cattle inoculated with classic BSE ( $P>0.05$ versus control) (Figure 4, I, J, and M). Retinas from cattle inoculated with atypical BSEs had significantly more ASC immunoreactivity (approximately eightfold increase), compared with the retinas from cattle in the sham or classic BSE inoculated groups (Figure 4, $\mathrm{K}-\mathrm{M}$ ). Assembly of the NLRP3 inflammasome triggers cleavage of procaspase- 1 into active caspase- 1 that converts the cytokine precursor pro-IL-1 $\beta$ into active IL-1 $\beta$, a key proinflammatory mediator. $^{39}$ Western blot analysis of downstream inflammasome markers, active caspase- 1 and IL-1 $\beta$, showed minimal protein expression in retinas from cattle in either the sham or classic BSE inoculation groups $(P>0.05)$ (Figure 4, N-P). In comparison, the retinas from cattle inoculated with atypical BSEs had a significant increase in both caspase- 1 and IL-1 $\beta$ protein expression (Figure 4, N-P).

Similar results were observed in the brainstem at the level of the obex, thalamus, and caudate nucleus (Supplemental Figures S3 and S4). Consistent with results from the retina, double immunofluorescence and confocal microscopy confirmed that NLRP3 was localized to $\mathrm{Iba}^{+}$ microglia in the obex (Supplemental Figure S3, A-C), thalamus (data not shown), and caudate nucleus (data not shown). The obex and thalami from cattle inoculated with BSE-H or BSE-L had significantly more NLRP3 
A

Neuroinflammation versus Incubation Time

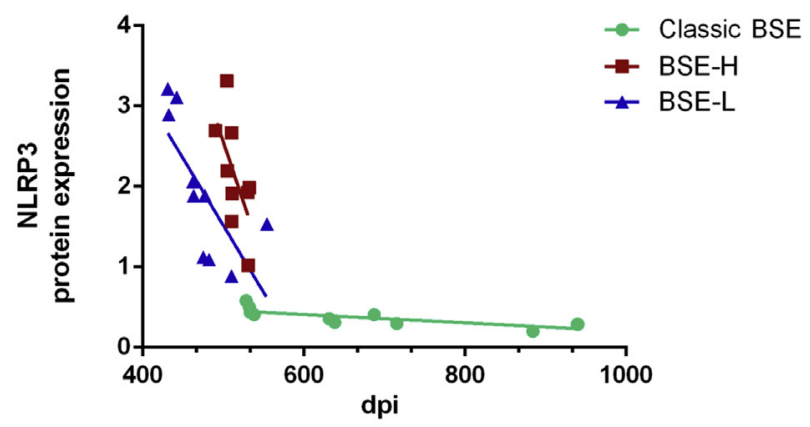

B

\section{Macroautophagy versus} Incubation Time

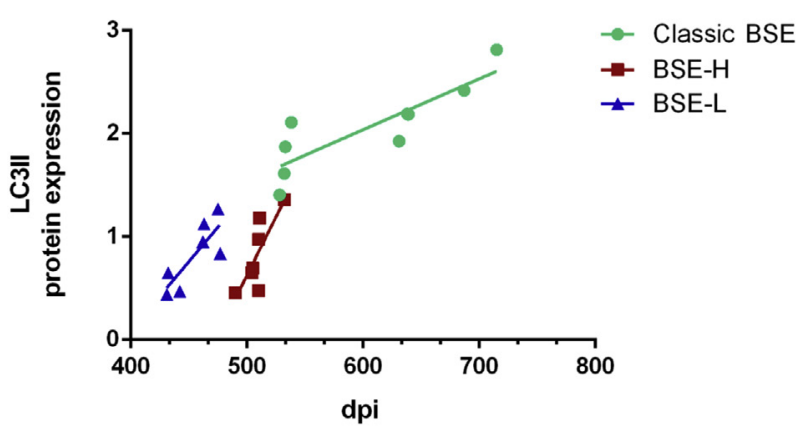

Figure 6 Reciprocal regulation between neuroinflammation and autophagy is reflected in incubation periods. A: Graph represents a negative relationship between disease incubation period and neuroinflammation (represented by percentage fluorescence of NLRP3 immunoreactivity, also shown in Figure 4 ) in retinas from cattle inoculated with classic bovine spongiform encephalopathy (BSE), BSE-H, or BSE-L. B: Graph represents a negative relationship between disease incubation period and autophagy (represented by LC3II protein expression, also shown in Figure 5) in retinas from cattle inoculated with classic BSE, BSE-H, or BSE-L. dpi, days post inoculation.

(Supplemental Figures S3, D and E) and ASC (Supplemental Figures S3, G and H) immunoreactivity compared with cattle inoculated with sham or classic BSE. Levels of NLRP3 and ASC immunolabeling in the caudate nucleus were comparable in cattle inoculated with classic BSE, BSE-H, or BSE-L (Supplemental Figures S3I). The obex and thalami from cattle inoculated with BSE-H and BSE-L also had higher expression of caspase-1 (Supplemental Figure S4, A, B, and D) and IL-1 $\beta$ (Supplemental Figure S4, A, C, and E), compared with cattle inoculated with sham or classic BSE. Protein expression levels of caspase-1 (Supplemental Figure S4, A and F) and IL-1 $\beta$ (Supplemental Figure S4, A and G) in the caudate nucleus were comparable in classic BSE, BSE-H, and BSE-L. These results demonstrate increased microglia-driven NALP3 inflammasome activation in cattle inoculated with atypical BSEs, that parallels increased $\operatorname{PrP}^{\mathrm{Sc}}$ accumulation and shorter disease incubation times.
Autophagic Progression Is Impaired in Atypical BSE-H and BSE-L

In protein misfolding diseases, an increase in autophagy is a proper response during clearing of bulk cytoplasmic contents. A decrease below basal levels may indicate autophagic dysfunction due to an overload of a system incapable of degrading proteins. ${ }^{40-43}$ To assess the relationship between incubation time and autophagy in the retina, immunohistochemical and Western blot analysis of proteins involved in the initiation of autophagy and vesicle formation was performed (Figure 5). To determine changes in vesicle formation, distribution and protein expression of the autophagosome marker, LC3, was analyzed. Retinas from cattle from the sham and classic BSE inoculation groups had diffuse LC3 immunoreactivity localized to the outer segments and synaptic layers, consistent with a basal level of autophagy under normal conditions (Figure 5A). Retinas from cattle inoculated with classic BSE had robust, multifocal LC3 immunoreactivity primarily in the synaptic layers, similar to the pattern of $\mathrm{PrP}^{\mathrm{Sc}}$ immunoreactivity in these cattle (Figure 5B). In contrast, retinas from cattle inoculated with atypical BSE-H and BSE-L had inappreciable and diffuse LC3 immunoreactivity, comparable to retinas from control cattle (Figure 5, C and D). Western blot analysis of LC3II protein expression revealed similar results. Compared with control, level of LC3II protein expression was approximately twofold higher in retinas from classic BSE cattle $(P>0.001)$, and comparable in retinas of BSE-H $(P>0.05)$ and BSE-L $(P>0.05)$ cattle (Figure 5, E and F).

To determine changes in the initiation of autophagy, the expression levels of the key autophagy-initiation protein, pULK1 ${ }^{\mathrm{S} 757}$, were examined. Briefly, the initiation stage involves activation of ULK1 (a serine-threonine kinase), regulated by both mammalian target of rapamycin and AMPK. Activation of mammalian target of rapamycin leads to the enzymatic inactivation of ULK1, by downstream phosphorylation at serine 757. Conversely, when 5' AMPactivated protein kinase is activated, ULK1 is dephosphorylated at serine 757 and thereby activated, allowing for autophagy to advance. ${ }^{44}$ Compared with control, protein levels of pULK $1{ }^{\mathrm{S} 757}$ were significantly lower in retinas from cattle inoculated with classic BSE (Figure 5, E and G). Protein levels of pULK1 ${ }^{\mathrm{S} 757}$ in retinas from cattle inoculated with BSE-H and BSE-L were comparable to basal levels detected in retinas from sham-inoculated cattle (Figure 5, E and G). Again, similar results were observed in the brainstem, thalamus, and caudate nucleus (Supplemental Figure S5). The brainstem, and thalami from cattle inoculated with BSE-H and BSE-L, had significantly higher expression of LC3II (Supplemental Figure S5, A, B, and D), and lower expression of pULK1 ${ }^{\mathrm{S} 757}$ (Supplemental Figure S5, A, C, and E). However, protein expression levels of LC3II (Supplemental Figure S5, A and F) and pULK1 $1^{\text {S757 }}$ (Supplemental Figure S5, A and G) in the caudate nucleus were similar in tissues from cattle inoculated with classic BSE, BSE-H, or 
BSE-L. These results indicate an inverse relationship between incubation period and autophagy, suggesting that autophagic dysfunction may contribute to increased $\operatorname{PrP}^{\mathrm{Sc}}$ accumulation in atypical BSEs, leading to an accelerated disease phenotype and shorter incubation periods.

Taken together, we report an inverse correlation between incubation time and NLRP3 inflammasome activation and a positive correlation between incubation period and autophagy (Figure 6). This work suggests a reciprocal regulation between $\operatorname{PrP}^{\mathrm{Sc}}$-associated neuroinflammation and autophagy in vivo that reflects differences in incubation period and disease progression.

\section{Discussion}

In this study, we report a relationship between straindependent differences in disease incubation period and accumulation of $\mathrm{PrP}^{\mathrm{Sc}}$ in the retina, brainstem, and thalamus. Specifically, cattle inoculated with the agents of atypical BSE-H or BSE-L had shorter incubation periods and greater areas of $\operatorname{PrP}^{\mathrm{Sc}}$ deposition compared with cattle inoculated with classic BSE, demonstrating a negative relationship between incubation period and disease pathology. Prion disease strains can be differentiated by incubation periods, neuroanatomic and cellular deposition patterns of $\mathrm{PrP}^{\mathrm{Sc}}$, and other molecular properties. ${ }^{5-8,45,46}$ Distinct prion strains, within a species, have been shown to exhibit different patterns and levels of $\operatorname{PrP}^{\mathrm{Sc}}$ accumulation in the brain independent of incubation period. For example, $\operatorname{PrP}^{\mathrm{Sc}}$ deposition in classic scrapie in sheep occurs primarily in the medulla oblongata rather than the cortices of the cerebellum and the cerebrum, as seen in atypical sheep scrapie. ${ }^{4,7,47}$ In addition, spongiform lesions and $\mathrm{PrP}^{\mathrm{Sc}}$ immunoreactivity in the brains from cattle inoculated with classic BSE are distinct from those of cattle with atypical BSE-H. In natural and experimental cases of classic BSE, spongiform lesions are distributed consistently throughout the brain, ${ }^{48}$ whereas in $\mathrm{BSE}-\mathrm{H}$, spongiform lesions and $\mathrm{PrP}^{\mathrm{Sc}}$ immunoreactivity are more intense in the brainstem and midbrain, increasing in intensity caudally, from the frontal cortex to the occipital cortex. ${ }^{16,36,48}$ Moreover, patterns of $\mathrm{PrP}^{\mathrm{Sc}}$ immunoreactivity (ie, intraglial, stellate, and intraneuronal) in the brains from BSE-H and classic BSE affected cattle vary depending on region. ${ }^{16,36}$ Although strain-dependent patterns of $\mathrm{PrP}^{\mathrm{Sc}}$ accumulation have been widely explored, studies examining the relationship between strain-dependent incubation period and extent of $\operatorname{PrP}^{\mathrm{Sc}}$ deposition are lacking.

Historically, glial activation has been assumed to be merely a response to pathophysiological events in proteinmisfolding diseases; however, more recent studies have established key contributions of glial cells to neuroinflammation and neurodegenerative disease pathogenesis. ${ }^{49}$ Although this study does not prove a causal relationship, this work describes a positive correlation between glial cell activation and $\operatorname{PrP}^{\mathrm{Sc}}$ accumulation that is reflected in disease incubation period. Compared with cattle with classic BSE, the retinas from cattle inoculated with atypical BSEs (BSE-H and BSE-L) had robust activation of Müller glia that paralleled intense $\mathrm{PrP}^{\mathrm{Sc}}$ accumulation and shorter incubation periods. Müller glia, which are normally quiescent, are ubiquitously activated in response to a wide range of pathogenic stimuli, exerting protective and toxic effects on retinal neurons. Although activation of Müller glia has been previously reported in BSE, ${ }^{13}$ and other prion diseases, ${ }^{12}$ the relationship between incubation period and Müller glial activation in the retina has not been previously described. Examination of several brain regions demonstrated that compared with classic BSE, the brainstems and thalami from cattle inoculated with atypical BSEs had more intense activation of astrocytes that paralleled $\mathrm{PrP}^{\mathrm{Sc}}$ accumulation. However, levels of astrocyte activation in caudate nuclei were comparable in classic and atypical BSEs. The close association of activated astrocytes and $\mathrm{PrP}^{\mathrm{Sc}}$ deposits is well documented in prion disease, further supporting these results. ${ }^{50-52}$

This study demonstrates an association between straindependent incubation period, $\operatorname{PrP}^{\mathrm{Sc}}$ accumulation, and microglial activation in vivo. These results show that compared with classic BSE, retinas from cattle inoculated with atypical BSEs had altered microglial morphology and robust activation that also paralleled intense $\mathrm{PrP}^{\mathrm{Sc}}$ accumulation and shorter incubation periods. A similar relationship was seen in the brainstem and thalamus, but not in the caudate nucleus. The molecular mechanisms underlying the microglial response to $\mathrm{PrP}^{\mathrm{Sc}}$ are still largely unknown. Previous work reported that the depletion of microglia resulted in enhanced $\mathrm{PrP}^{\mathrm{Sc}}$ accumulation and augmented prion infectivity, suggesting a neuroprotective role. ${ }^{53}$ Several time course studies reported that the onset of microglial activation occurs at an early preclinical phase of prion disease, before spongiform change and/or neuronal loss, indicating that microglia may contribute to $\mathrm{PrP}^{\mathrm{Sc}}$ induced neurodegeneration. ${ }^{12,54-56}$ The current study shows that the distribution of microglial response positively correlates with $\mathrm{PrP}^{\mathrm{Sc}}$ deposition, indicating a negative relationship between incubation period and microglial activation. Several murine models of prion disease describe the role of microglia in prion pathogenesis, highlighting shortened or prolonged disease incubation period and augmented $\operatorname{PrP}^{\mathrm{Sc}}$ accumulation as a common effect of modulating microglia-related molecules (ie, toll-like receptors, cytokines and chemokines, and complement systems). ${ }^{57}$ The detrimental effects of microglial activation in prion disease also were investigated in the context of NALP3 inflammasome activation. ${ }^{21,22}$ The NALP3 inflammasome is a multiprotein complex that has been shown to be activated by PrP fibrils, leading to the secretion of IL- $1 \beta$ and other proinflammatory factors. ${ }^{21,23-25}$ These results demonstrate robust NLRP3 inflammasome activation in the retinas from cattle inoculated with BSE-H or BSE-L, as evidenced by a significant up-regulation of key 
inflammasome proteins (ie, NLRP, ASC, active caspase-1, and active IL-1 $\beta$ ). Retinas from cattle inoculated with classic BSE had minimal NLRP3 and ASC immunoreactivity, whereas protein expression levels of downstream inflammatory mediators, caspase- 1 and IL- $1 \beta$, were comparable to controls. Although previous research has identified several independent mechanisms at play in regard to NALP3 inflammasome activation in response to PrP fibrils, this is the first report that describes the relationship between incubation period, $\operatorname{PrP}^{\mathrm{Sc}}$ accumulation, and NLRP3 inflammasome activation in a natural host of prion disease. ${ }^{21,22,58}$

This work describes contrasting events in the autophagic pathway: up-regulation of autophagy in the retinas from cattle inoculated with classic BSE, indicated by the increase in LC3II, decrease in pULK1 ${ }^{\mathrm{S} 757}$, and down-regulation of autophagy in the retinas from cattle inoculated with BSE-H and BSE-L. Recently, it was reported that the inhibition of autophagy enhances release of active IL-1 $\beta$ and, inversely, gene silencing of the NALP3 inflammasome promotes autophagy in an in vitro prion model. ${ }^{26}$ In the current study, up-regulation of autophagy in classic BSE paralleled longer incubation periods (approximately 640 days postinoculation). Conversely, down-regulation of autophagy correlated with robust $\mathrm{PrP}^{\mathrm{Sc}}$ accumulation, NLRP3 inflammasome activation, and shorter incubation periods (approximately 490 days postinoculation). The neuroprotective role of autophagy is suggested by several studies, demonstrating that the up-regulation of autophagic flux can clear misfolded and aggregated intracellular proteins and suppress neuroinflammation via the degradation of IL- $1 \beta .^{41,59-67}$ Although numerous studies show the up-regulation of autophagosomes in response to misfolded proteins in neurodegenerative diseases (ie, prion disease, $\mathrm{PD}$, and $\mathrm{AD}$ ), whether this event is a proper response to accumulation of misfolded proteins or due to an impairment in the degradation of autophagosomes is not known. ${ }^{34}$ Furthermore, it is unknown whether an impairment in the degradation of misfolded proteins is yet another factor that leads to protein aggregation or whether autophagic dysfunction is driven by misfolded proteins. This work demonstrates an inverse relationship between neuroinflammation and autophagy in vivo that is strain dependent and is reflected in disease incubation period. In line with our work, a recent study investigated autophagic regulation at different stages of scrapie in ovinized $\operatorname{Tg} 338$ mice and reported that autophagic dysregulation may be part of the pathologic process of scrapie, evident in the late stage of prion infection. ${ }^{68}$

A hallmark of prion diseases and other neurodegenerative proteinopathies is the formation of misfolded protein aggregates that cause neuroinflammation and cellular toxicity that contribute to cellular collapse. Therapeutic options that are being explored target different steps in the synthesis, processing, and degradation of proteins implicated in neurodegenerative diseases. A major challenge that impacts the development of therapies is the incomplete knowledge of factors that influence disease progression. The straindependent variation in incubation periods between classic and atypical BSEs provides a unique platform to investigate the molecular underpinnings that lead to an accelerated disease progression in atypical BSEs compared with classic BSE. Herein, we describe the relationship between incubation period, $\mathrm{PrP}^{\mathrm{Sc}}$ accumulation, microglia-driven NLRP3 inflammasome activation, and autophagy that has not been previously reported in natural host of prion disease. Targeting potential mechanisms of several NLRP3 inflammasome inhibitors has been increasingly regarded as an effective approach to developing therapies for $\mathrm{PD}, \mathrm{AD}$, and Huntington disease. ${ }^{69-73}$ Inhibition of macroautophagy is widely shown to be a contributing factor in several proteinopathies; therefore, up-regulation of autophagy has also been regarded as a valuable therapeutic strategy. ${ }^{74}$ The roles of gliosis, the NLRP3 inflammasome, and protein clearance via macroautophagy all have been individually studied in the pathogenesis and progression of several protein misfolding diseases, including prion disease, $\mathrm{PD}, \mathrm{AD}$, and Huntington disease. ${ }^{69-73}$ This study provides a unique framework for understanding an interconnected system in which the progression of protein misfolding disease is affected by the amount of misfolded proteins in the tissue, the corresponding neuroinflammatory response, and the protein-clearance system.

Collectively, this study describes several key relationships between strain-dependent incubation period, $\mathrm{PrP}^{\mathrm{Sc}}$ accumulation, neuroinflammation, and the autophagic stress response. A caveat of this study is that it does not investigate the role of differing amounts of infectivity in the three BSE isolates; therefore, it is possible that these observations are in part due to the amount of infectivity in the inocula and not just the inherent biological potency of each strain. We demonstrate that the retinas from cattle inoculated with atypical BSEs (BSE-H and BSE-L), characterized with shorter incubation periods compared with classic BSE, have robust $\operatorname{PrP}^{\mathrm{Sc}}$ accumulation. Greater $\mathrm{PrP}^{\mathrm{Sc}}$ accumulation in these cattle corresponds to significantly increased retinal glial cell activation and microglia-driven NRLP3 inflammasome activation that is also reflected in a shorter incubation period. Finally, increased $\mathrm{PrP}^{\mathrm{Sc}}$ accumulation and NLRP3 activation coincide with the down-regulation of autophagy and shorter incubation periods. These findings support and further clarify a previously reported mechanism describing the interconnection of neuroinflammation and autophagy in response to prion disease. ${ }^{26} \mathrm{We}$ describe the relationship between strain-dependent misfolded protein accumulation, neuroinflammation, and autophagy in vivo in a natural host of prion disease that may be predictors of disease incubation period. These results should advance our understanding of the interaction between disease pathogenesis and prion strain-dependent incubation periods and help identify new potential therapeutic targets for intervention. 


\section{Acknowledgments}

We thank Adrienne Shircliff, Judith Stasko, Simon Moe, Trevor Buhr, and Robyn D. Kokemuller for technical assistance.

\section{Supplemental Data}

Supplemental material for this article can be found at http://doi.org/10.1016/j.ajpath.2020.03.006.

\section{References}

1. Sweeney P, Park H, Baumann M, Dunlop J, Frydman J, Kopito R, McCampbell A, Leblanc G, Venkateswaran A, Nurmi A: Protein misfolding in neurodegenerative diseases: implications and strategies. Transl Neurodegener 2017, 6:6

2. Kupfer L, Hinrichs W, Groschup M: Prion protein misfolding. Curr Mol Med 2009, 9:826-835

3. Morales R, Green KM, Soto C: Cross currents in protein misfolding disorders: interactions and therapy. CNS Neurol Disord Drug Targets $2009,8: 363-371$

4. Greenlee JJ, Greenlee MHW: The transmissible spongiform encephalopathies of livestock. ILAR J 2015, 56:7-25

5. Fraser H, Dickinson A: The sequential development of the brain lesions of scrapie in three strains of mice. J Comp Pathol 1968, 78: $301-311$

6. Fraser H, Dickinson A: Scrapie in mice: agent-strain differences in the distribution and intensity of grey matter vacuolation. J Comp Pathol 1973, 83:29-40

7. Gonzalez L, Martin S, Begara-McGorum I, Hunter N, Houston F, Simmons M, Jeffrey M: Effects of agent strain and host genotype on $\operatorname{PrP}$ accumulation in the brain of sheep naturally and experimentally affected with scrapie. J Comp Pathol 2002, 126:17-29

8. Bruce ME: TSE strain variation: an investigation into prion disease diversity. Br Med Bull 2003, 66:99-108

9. Bessen RA, Marsh RF: Identification of two biologically distinct strains of transmissible mink encephalopathy in hamsters. J Gen Virol 1992, 73:329-334

10. Ayers JI, Schutt CR, Shikiya RA, Aguzzi A, Kincaid AE, Bartz JC: The strain-encoded relationship between PrPSc replication, stability and processing in neurons is predictive of the incubation period of disease. PLoS Pathog 2011, 7:e1001317

11. Carroll JA, Striebel JF, Rangel A, Woods T, Phillips K, Peterson KE, Race B, Chesebro B: Prion strain differences in accumulation of PrPSc on neurons and glia are associated with similar expression profiles of neuroinflammatory genes: comparison of three prion strains. PLoS Pathog 2016, 12:e1005551

12. Greenlee MHW, Lind M, Kokemuller R, Mammadova N, Kondru N, Manne S, Smith J, Kanthasamy A, Greenlee J: Temporal resolution of misfolded prion protein transport, accumulation, glial activation, and neuronal death in the retinas of mice inoculated with scrapie. Am J Pathol 2016, 186:2302-2309

13. Greenlee MHW, Smith JD, Platt EM, Juarez JR, Timms LL, Greenlee JJ: Changes in retinal function and morphology are early clinical signs of disease in cattle with bovine spongiform encephalopathy. PLoS One 2015, 10:e119431

14. Hamir A, Miller J, Kunkle R, Hall S, Richt J: Susceptibility of cattle to first-passage intracerebral inoculation with chronic wasting disease agent from white-tailed deer. Vet Pathol 2007, 44:487-493

15. Greenlee J, Hamir A, Greenlee MW: Abnormal prion accumulation associated with retinal pathology in experimentally inoculated scrapie-affected sheep. Vet Pathol 2006, 43:733-739
16. Moore SJ, West Greenlee MH, Smith JD, Vrentas CE, Nicholson EM, Greenlee JJ: A comparison of classical and H-type bovine spongiform encephalopathy associated with E211K prion protein polymorphism in wild-type and EK211 cattle following intracranial inoculation. Front Vet Sci 2016, 3:78

17. Spraker T, O’Rourke KI, Gidlewski T, Powers J, Greenlee J, Wild M: Detection of the abnormal isoform of the prion protein associated with chronic wasting disease in the optic pathways of the brain and retina of Rocky Mountain elk (Cervus elaphus nelsoni). Vet Pathol 2010, 47:536-546

18. Hortells P, Monzón M, Monleón E, Acín C, Vargas A, Bolea R, Luján L, Badiola JJ: Pathological findings in retina and visual pathways associated to natural Scrapie in sheep. Brain Res 2006, 1108:188-194

19. Smith J, Greenlee J, Hamir A, Richt J, Greenlee MW: Retinal function and morphology are altered in cattle infected with the prion disease transmissible mink encephalopathy. Vet Pathol 2009, 46:810-816

20. Orrù $\mathrm{CD}$, Soldau K, Cordano C, Llibre-Guerra J, Green AJ, Sanchez H, Groveman BR, Edland SD, Safar JG, Lin JH: Prion seeds distribute throughout the eyes of sporadic Creutzfeldt-Jakob disease patients. mBio 2018, 9:e02095-18

21. Shi F, Yang L, Kouadir M, Yang Y, Wang J, Zhou X, Yin X, Zhao D: The NALP3 inflammasome is involved in neurotoxic prion peptideinduced microglial activation. J Neuroinflammation 2012, 9:73

22. Shi F, Kouadir M, Yang Y: NALP3 inflammasome activation in protein misfolding diseases. Life Sci 2015, 135:9-14

23. Hafner-Bratkovič I, Benčina M, Fitzgerald KA, Golenbock D, Jerala R: NLRP3 inflammasome activation in macrophage cell lines by prion protein fibrils as the source of IL-1 $\beta$ and neuronal toxicity Cell Mol Life Sci 2012, 69:4215-4228

24. Tribouillard-Tanvier D, Striebel JF, Peterson KE, Chesebro B Analysis of protein levels of 24 cytokines in scrapie agent-infected brain and glial cell cultures from mice differing in prion protein expression levels. J Virol 2009, 83:11244-11253

25. Shi F, Yang Y, Kouadir M, Fu Y, Yang L, Zhou X, Yin X, Zhao D Inhibition of phagocytosis and lysosomal acidification suppresses neurotoxic prion peptide-induced NALP3 inflammasome activation in BV2 microglia. J Neuroimmunol 2013, 260:121-125

26. Lai M, Yao H, Shah SZA, Wu W, Wang D, Zhao Y, Wang L, Zhou X, Ming ZD, Yang L: The NLRP3-caspase 1 inflammasome negatively regulates autophagy via TLR4-TRIF in prion peptideinfected microglia. Front Aging Neurosci 2018, 10:116

27. Salminen A, Kaarniranta K, Kauppinen A: Inflammaging: disturbed interplay between autophagy and inflammasomes. Aging (Albany NY) 2012, 4:166

28. Committee for the Update of the Guide for the Care and Use of Laboratory AnimalsNational Research Council: Guide for the Care and Use of Laboratory Animals: Eighth Edition. Washington, DC, National Academies Press, 2011

29. Greenlee JJ, Nicholson EM, Smith JD, Kunkle RA, Hamir AN: Susceptibility of cattle to the agent of chronic wasting disease from elk after intracranial inoculation. J Vet Diagn Invest 2012, 24:1087-1093

30. Richt JA, Kunkle RA, Alt D, Nicholson EM, Hamir AN, Czub S, Kluge J, Davis AJ, Hall SM: Identification and characterization of two bovine spongiform encephalopathy cases diagnosed in the United States. J Vet Diagn Invest 2007, 19:142-154

31. Vrentas CE, Greenlee JJ, Baron T, Caramelli M, Czub S, Nicholson EM: Stability properties of PrP Sc from cattle with experimental transmissible spongiform encephalopathies: use of a rapid whole homogenate, protease-free assay. BMC Vet Res 2013, 9:167

32. Hamir A, Kunkle R, Miller J, Bartz JC, Richt J: First and second cattle passage of transmissible mink encephalopathy by intracerebral inoculation. Vet Pathol 2006, 43:118-126

33. Hamir A, Kunkle R, Miller J, Greenlee J, Richt J: Experimental second passage of chronic wasting disease (CWDmuledeer) agent to cattle. J Comp Pathol 2006, 134:63-69

34. Mammadova N, Summers CM, Kokemuller RD, He Q, Ding S, Baron T, Yu C, Valentine RJ, Sakaguchi DS, Kanthasamy AG: 
Accelerated accumulation of retinal $\alpha$-synuclein (pSer129) and tau, neuroinflammation, and autophagic dysregulation in a seeded mouse model of Parkinson's disease. Neurobiol Dis 2019, 121:1-16

35. Jensen EC: Quantitative analysis of histological staining and fluorescence using ImageJ. Anat Rec 2013, 296:378-381

36. Greenlee JJ, Smith JD, Greenlee MHW, Nicholson EM: Clinical and pathologic features of $\mathrm{H}$-type bovine spongiform encephalopathy associated with E211K prion protein polymorphism. PLoS One 2012, 7:e38678

37. Bringmann A, Pannicke T, Grosche J, Francke M, Wiedemann P, Skatchkov SN, Osborne NN, Reichenbach A: Müller cells in the healthy and diseased retina. Prog Retin Eye Res 2006, 25:397-424

38. Walsh JG, Muruve DA, Power C: Inflammasomes in the CNS. Nat Rev Neurosci 2014, 15:84

39. Martinon F, Burns K, Tschopp J: The inflammasome: a molecular platform triggering activation of inflammatory caspases and processing of proIL- $\beta$. Mol Cell 2002, 10:417-426

40. Klionsky DJ, Abdelmohsen K, Abe A, Abedin MJ, Abeliovich H, Acevedo Arozena A, Adachi H, Adams CM, Adams PD, Adeli K: Guidelines for the use and interpretation of assays for monitoring autophagy. Autophagy 2016, 12:1-222

41. Ishibashi D, Homma T, Nakagaki T, Fuse T, Sano K, Takatsuki H, Atarashi R, Nishida N: Strain-dependent effect of macroautophagy on abnormally folded prion protein degradation in infected neuronal cells. PLoS One 2015, 10:e137958

42. Liberski PP: Cell death and autophagy in prion diseases. Prion Diseases. San Francisco, CA: Springer, 2017. pp. 145-158

43. Milisav I, Šuput D, Ribarič S: Unfolded protein response and macroautophagy in Alzheimer's, Parkinson's and prion diseases. Molecules 2015, 20:22718-22756

44. Jaeger PA, Wyss-Coray T: All-you-can-eat: autophagy in neurodegeneration and neuroprotection. Mol Neurodegener 2009, 4:16

45. Bruce M, McBride P, Farquhar C: Precise targeting of the pathology of the sialoglycoprotein, PrP, and vacuolar degeneration in mouse scrapie. Neurosci Lett 1989, 102:1-6

46. Bartz JC: Prion strain diversity. Cold Spring Harb Perspect Med 2016, 6:a024349

47. Greenlee JJ: Update on classical and atypical scrapie in sheep and goats. Vet Pathol 2019, 56:6-16

48. Okada $\mathrm{H}$, Iwamaru $\mathrm{Y}$, Imamura $\mathrm{M}$, Masujin $\mathrm{K}$, Matsuura $\mathrm{Y}$, Shimizu Y, Kasai K, Mohri S, Yokoyama T, Czub S: Experimental Htype bovine spongiform encephalopathy characterized by plaques and glial- and stellate-type prion protein deposits. Vet Res 2011, 42:79

49. Takeuchi H: Roles of glial cells in neuroinflammation and neurodegeneration. Clin Exp Neuroimmunol 2013, 4:2-16

50. Brambilla L, Martorana F, Rossi D: Astrocyte signaling and neurodegeneration: new insights into CNS disorders. Prion 2013, 7:28-36

51. Hernández RS, Sarasa R, Toledano A, Badiola JJ, Monzón M: Morphological approach to assess the involvement of astrocytes in prion propagation. Cell Tissue Res 2014, 358:57-63

52. Sarasa R, Martínez A, Monleón E, Bolea R, Vargas A, Badiola JJ, Monzón M: Involvement of astrocytes in transmissible spongiform encephalopathies: a confocal microscopy study. Cell Tissue Res 2012, 350:127-134

53. Falsig J, Julius C, Margalith I, Schwarz P, Heppner FL, Aguzzi A: A versatile prion replication assay in organotypic brain slices. Nat Neurosci 2008, 11:109

54. Betmouni S, Perry V, Gordon J: Evidence for an early inflammatory response in the central nervous system of mice with scrapie. Neuroscience 1996, 74:1-5

55. Giese A, Brown DR, Groschup MH, Feldmann C, Haist I, Kretzschmar HA: Role of microglia in neuronal cell death in prion disease. Brain Pathol 1998, 8:449-457
56. Williams A, Lucassen P, Ritchie D, Bruce M: PrP deposition, microglial activation, and neuronal apoptosis in murine scrapie. Exp Neurol 1997, 144:433-438

57. Aguzzi A, Zhu C: Microglia in prion diseases. J Clin Invest 2017 , 127:3230-3239

58. Nuvolone M, Sorce S, Schwarz P, Aguzzi A: Prion pathogenesis in the absence of NLRP3/ASC inflammasomes. PLoS One 2015, 10: e0117208

59. Rubinsztein DC, Gestwicki JE, Murphy LO, Klionsky DJ: Potential therapeutic applications of autophagy. Nat Rev Drug Discov 2007, 6: 304

60. Martinez-Vicente M, Cuervo AM: Autophagy and neurodegeneration: when the cleaning crew goes on strike. Lancet Neurol 2007, 6:352-361

61. Saitoh T, Fujita N, Jang MH, Uematsu S, Yang B-G, Satoh T, Omori H, Noda T, Yamamoto N, Komatsu M: Loss of the autophagy protein Atg16L1 enhances endotoxin-induced IL-1 $\beta$ production. Nature 2008, 456:264

62. Jo E-K, Shin D-M, Choi AM: Autophagy: cellular defense to excessive inflammation. Microbes Infect 2012, 14:119-125

63. Harris J, Hartman M, Roche C, Zeng SG, O'Shea A, Sharp FA, Lambe EM, Creagh EM, Golenbock DT, Tschopp J: Autophagy controls IL-1 $\beta$ secretion by targeting pro-IL-1 $\beta$ for degradation. J Biol Chem 2011, 286:9587-9597

64. Nakahira K, Haspel JA, Rathinam VA, Lee S-J, Dolinay T, Lam HC, Englert JA, Rabinovitch M, Cernadas M, Kim HP: Autophagy proteins regulate innate immune response by inhibiting NALP3 inflammasome-mediated mitochondrial DNA release. Nat Immunol 2011, 12:222

65. Joshi-Barr S, Bett C, Chiang W-C, Trejo M, Goebel HH, Sikorska B, Liberski P, Raeber A, Lin JH, Masliah E: De novo prion aggregates trigger autophagy in skeletal muscle. J Virol 2014, 88:2071-2082

66. Goold R, McKinnon C, Tabrizi SJ: Prion degradation pathways: potential for therapeutic intervention. Mol Cell Neurosci 2015, 66: $12-20$

67. Abdelaziz DH, Thapa S, Abdulrahman B, Vankuppeveld L, Schatzl HM: Metformin reduces prion infection in neuronal cells by enhancing autophagy. Biochem Biophys Res Commun 2020, 523: 423-428

68. López-Pérez Ó, Toivonen JM, Otero A, Solanas L, Zaragoza P, Badiola JJ, Osta R, Bolea R, Martín-Burriel I: Impairment of autophagy in scrapie-infected transgenic mice at the clinical stage. Lab Invest 2019, 100:52-63

69. Wang H-M, Zhang T, Huang J-K, Xiang J-Y, Chen J-j, Fu J-L, Zhao Y-W: Edaravone attenuates the proinflammatory response in amyloid- $\beta$-treated microglia by inhibiting NLRP3 inflammasomemediated IL-1 $\beta$ secretion. Cell Physiol Biochem 2017, 43: $1113-1125$

70. Falcão AS, Carvalho LA, Lidónio G, Vaz AR, Lucas SD, Moreira R, Brites D: Dipeptidyl vinyl sulfone as a novel chemical tool to inhibit HMGB1/NLRP3-inflammasome and inflamma-miRs in A $\beta$-mediated microglial inflammation. ACS Chem Neurosci 2016, 8:89-99

71. Li D, Yang H, Ma J, Luo S, Chen S, Gu Q: MicroRNA-30e regulates neuroinflammation in MPTP model of Parkinson's disease by targeting Nlrp3. Hum Cell 2018, 31:106-115

72. Fan Z, Liang Z, Yang H, Pan Y, Zheng Y, Wang X: Tenuigenin protects dopaminergic neurons from inflammation via suppressing NLRP3 inflammasome activation in microglia. J Neuroinflammation 2017, 14:256

73. Sperlágh B, Illes P: P2X7 receptor: an emerging target in central nervous system diseases. Trends Pharmacol Sci 2014, 35:537-547

74. Menzies FM, Moreau K, Rubinsztein DC: Protein misfolding disorders and macroautophagy. Curr Opin Cell Biol 2011, 23:190-197 